\title{
Exploring Attitudes and Reactions to Unfamiliar Food Pairings: An Examination of the Underlying Motivations and the Impact of Culinary Education
}

\author{
Mark Traynor \\ Auburn University, Montgomery, mark.traynor@auburn.edu \\ Andrew Moreo \\ Florida International University, a.moreo@fiu.edu \\ Lisa Cain \\ Florida International University, Icain@fiu.ie
}

See next page for additional authors

Follow this and additional works at: https://arrow.tudublin.ie/tfschafart

Part of the Food Chemistry Commons, and the Other Food Science Commons

\section{Recommended Citation}

Mark Traynor, Andrew Moreo, Lisa Cain, Roisin Burke \& Catherine Barry-Ryan (2020) Exploring Attitudes and Reactions to Unfamiliar Food Pairings: An Examination of the Underlying Motivations and the Impact of Culinary Education, Journal of Culinary Science \& Technology, DOI: 10.1080/15428052.2020.1732253

This Article is brought to you for free and open access by the School of Culinary Arts and Food Technology at ARROW@TU Dublin. It has been accepted for inclusion in Articles by an authorized administrator of ARROW@TU Dublin. For more information, please contact arrow.admin@tudublin.ie, aisling.coyne@tudublin.ie, gerard.connolly@tudublin.ie.

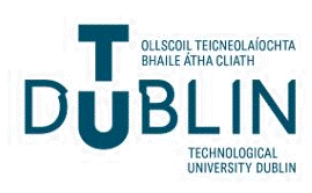




\section{Authors}

Mark Traynor, Andrew Moreo, Lisa Cain, Roisin Burke, and Catherine Barry-Ryan

This article is available at ARROW@TU Dublin: https://arrow.tudublin.ie/tfschafart/208 


\title{
Exploring Attitudes and Reactions to Unfamiliar Food Pairings: An Examination of the Underlying Motivations and the Impact of Culinary Education
}

\author{
Mark Traynor, Andrew Moreo, Lisa Cain, Roisin Burke, and Catherine Barry-Ryan \\ aDepartment of Nutrition, Dietetics, and Hospitality Management, Auburn University, Auburn, \\ Alabama, USA; ${ }^{b}$ Chaplin School of Hospitality and Tourism Management, Florida International \\ University, Miami, Alabama, USA; 'School of Culinary Arts and Food Technology, Technological \\ University Dublin, Dublin, Ireland; dSchool of Food Science and Environmental Health, Technological \\ University Dublin, Dublin, Ireland
}

\begin{abstract}
A mixed-methodology study was conducted to better understand consumer attitudes and behaviors toward novel food pairings and the impact of culinary education. Focus groups were conducted to investigate the underlying motivational factors to the reactions and behaviors toward unfamiliar foods. The second phase of the study consisted of sensory evaluation by two separate cohorts, panelists with and without culinary education, of food products created through the novel pairings of foods. Panelists with culinary education expressed a greater overall liking for the animal-based pairing. Sensory-Affective and Ideational factors appeared to be underlying motivational factors of these hedonic reactions.
\end{abstract}

\section{ARTICLE HISTORY}

Received 19 November 2019

Revised $\mathrm{xx}$ xxx xxxx

Accepted 16 February 2020

\section{KEYWORDS}

Food pairing; food behavior; unfamiliar foods; food reactions; food acceptance

\section{Introduction}

The food industry as a whole is a highly competitive and increasingly globalized environment. Establishing and sustaining a competitive advantage through product innovation is imperative for business growth and survival (Ottenbacher \& Harrington, 2009). A common approach for product innovation and differentiation amongst restaurant chefs and food product developers is the exploration of new and exciting food combinations (pairings). One of the foremost guiding principles for creating such food pairings focuses on combining the flavors of each food to create a single harmonious flavor profile (Varshney, Varshney, Wang, \& Myers, 2013). However, identifying and developing optimal food combinations that are received favorably by consumers is a challenging proposition for many food professionals (de Klepper, 2011). This experimentation with foods by chefs and manufacturers, driven by the desire to produce interesting and innovative foods, has led to an influx of innovative food products with novel flavor 
profiles in the marketplace. Thus, consumers encounter novel and unfamiliar food products on a regular basis (Stolzenbach, Byrne, \& Bredie, 2011). However, human acceptance of and preference for novel food products is a complex matter. Virtually all food preferences are learned, rather than innate (Gibson \& Brunstrom, 2007). Many novel products are met with negative reactions from consumers, such as disgust, resulting in a reluctance to consume the novel food and a relatively high failure rate (Barrena \& Sánchez, 2012). Thus, a common objective among food behavior researchers is to gain a better understanding of consumer reactions to foods and ultimately reduce the level of negative consumer reactions (Asperin, WooMi, \& Wolfe, 2011).

\section{Background}

\section{Food acceptance/rejection}

A framework for understanding the underlying reactions to both familiar and unfamiliar foods originates from a general taxonomy of basic motivation for food acceptance and rejection put forward by Rozin and Fallon (1987). This framework consists of three fundamental motivational dimensions: SensoryAffective, Anticipated Consequences, and Ideational. Each dimension is bipolar, with food substances near the negative poles being rejected, and foods near the positive poles being accepted (Martins, Pelchat, \& Pliner, 1997). For the SensoryAffective reactions dimension, foods are primarily accepted or rejected based on the hedonic response to their intrinsic sensory properties, such as taste, flavor, smell, or appearance (Martins \& Pliner, 2006). For instance, items with good taste (sensory properties that are positively perceived) are accepted, and food items with bad taste (sensory properties that are negatively perceived) are rejected (Rozin \& Fallon, 1986). Sensory-Affective reactions typically play a central role in food choice and acceptance decisions (Tuorila, 2007).

When assessing unfamiliar foods, Sensory-Affective motivations alone can be insufficient for understanding the consumers' reactions (attitudes and behaviors) toward foods (Martins \& Pliner, 2005). The second dimension, Anticipated Consequence, is centered on expectations concerning the expected consequences of ingesting a food item. These consequences can be either short or long term, provide potential health and/or pleasure benefits, or, be harmful in nature (Martins \& Pliner, 2006).

The final dimension, Ideational motivations, pertains to the knowledge of the nature or origin of the food. Foods considered closer to the positive poles of this dimension are thought of as being appropriate to consume. These foods have been transvalued positively; in the context of their cultural and social history, they are considered to be spiritually positive foods, and the consumption of such foods may transfer positive spiritual properties to the eater (Martins \& Pliner, 2005). 
The negative poles of Ideational motivations can be categorized by either disgust or inappropriateness (Ammann, Hartmann, \& Siegrist, 2018a). Rejection based on disgust is primarily motivated by the origin of the food (e.g., animal origin), what the food is (e.g., offal), and the social history of food preparation and handling in particular cultures (e.g., food prepared or handled by members of a lower class of a society particularly when the social norms of that society employ a caste system) (Rozin \& Fallon, 1987). This dimension can also be culturally bound, where food may be appropriate or desirable to one culture while evoking disgust in another culture, like Asian inspired meatballs that are comprised of a number of small animal byproducts instead of cow's meat (Martins \& Pliner, 2005). Unfamiliar foods of animal origin are known to be rejected more frequently than those of nonanimal origin (Pliner \& Pelchat, 1991).

\section{The impact of culinary education}

Concerning lessening one's propensity toward the negative poles of these dimensions, food education programs, primarily food sensory education, have been found to influence reactions and preferences (Hoppu, Prinz, Ojansivu, Laaksonen, \& Sandell, 2015; Mustonen, Rantanen, \& Tuorila, 2008; Mustonen \& Tuorila, 2010; Park \& Cho, 2016; Reverdy, Chesnel, Schlich, Köster, \& Lange, 2008; Reverdy, Schlich, Köster, Ginon, \& Lange, 2010). In particular, exposing people to diverse and unfamiliar foods through explicit food sensory education programs has shown to increase overall liking of novel foods and willingness to try unfamiliar foods in school-aged children participants (Mustonen \& Tuorila, 2010). Some researchers have noted that food sensory education programs can mitigate negative responses (reduce food neophobia scores) to unfamiliar foods for school-aged children (8-10 years) by enhancing the access and exposure to various more complex stimuli (Reverdy et al., 2008, 2010). Furthermore, the cognitive aspects of this education (providing a better vocabulary surrounding food) have the potential to support vibrant food discussion, thus making the students more consciously interested in food in general (Reverdy et al., 2010). These findings show that education around food can result in a significant decrease in food neophobia, and an increase in willingness to try novel foods in school-aged children.

\section{Research design and objectives}

A plethora of research publications have been devoted to understanding preference and acceptance toward familiar and unfamiliar foods and flavors (Backstrom, Pirttila-Backman, \& Tuorila, 2003, 2004; Chung et al., 2012; Henriques, King, \& Meiselman., 2009; Schickenberg, van Assema, Brug, \& De Vries, 2008). The vast majority of studies in this area investigated school-aged children's' attitudes and behaviors toward foods, with a limited number of 
studies exploring adult food preferences and responses (Ammann et al., 2018a; Ammann, Hartmann, \& Siegrist, 2018b; Elkins \& Zickgraf, 2018; Jaeger, Rasmussen, \& Prescott, 2017; King, Meiselman., \& Henriques, 2008; Knaapila et al., 2011; Tuorila, Lähteenmäki, Pohjalainen, \& Lotti, 2001). Only a handful of studies investigated the impact of food education programs on reactions and preferences toward unfamiliar foods (Hoppu et al., 2015; Mustonen, Rantanen, \& Tuorila, 2009; Park \& Cho, 2016; Reverdy et al., 2008; Reverdy, Lange, \& Schlich, 2004; Reverdy et al., 2010). More specifically, these studies investigate the impact of sensory education programs consisting of 12 lessons (one and a half-hour in length each), during which students are exposed to unfamiliar foods, and learn about the role of the human senses in the perception of food. In contrast, students enrolled in university-level culinary programs are exposed to more in-depth education on culinary arts - gastronomy, the professional cooking of foods, and the development and pairing of flavors.

This current exploratory study sought to further the understanding of the role of culinary education on the attitudes and reactions among adults toward unfamiliar food pairings. To do so, a mixed-methods approach, known as a sequential exploratory design, was used. This consisted of two distinct phases; qualitative research followed by quantitative research (Creswell, Plano Clark, Gutmann, \& Hanson, 2003). As is the intent of exploratory research designs, the first method (qualitative) was designed to inform the second method (quantitative) (Greene, Caracelli, \& Graham, 1989).

The first phase of the study consisted of qualitative research with the aim of exploring the attitudes and behaviors of participants toward unfamiliar food pairings. This aim was accomplished using three focus groups, during which the participants were exposed to novel odor stimuli (aroma pairings) of both animal and non-animal origin. A consensus among focus group participants regarding the preferred aroma pairings was established and served as the basis for the development of suitable food products for the second phase of the study. Subsequently, two novel ice-cream products were developed, one product pairing consisted of main flavor ingredients of animal origin, while the other contained non-animal origin flavor ingredients (hereafter, samples are referred to animal and non-animal samples).

The second phase of the study consisted of quantitative research investigating the impact of culinary education on the overall liking of the two novel food products. Hedonic sensory evaluation tests were carried out on these products to measure the reactions of two distinctive cohorts; a culinaryeducated cohort and a general cohort (control). The findings of the focus groups regarding the underlying motivation factors for food acceptance and rejection of foods were used to better understand the findings from the second phase of the study. The two hypotheses tested in this phase of the study were: 
i. H1: A culinary-educated cohort will like a novel animal ice cream product significantly more than a control cohort;

ii. H2: A culinary-educated cohort will like a novel non-animal ice cream product significantly more than a control cohort.

\section{Materials and methods}

The setting for the current research was in the country of Ireland. This Northern Atlantic island has a relatively distinctive cuisine influenced by the interaction of climate, geographic location, geology, tradition, conquest and colonization, and commerce (Mac con Iomaire, 2011). There is a considerably conservative trend in the eating habits of Irish people, with many adhering to traditional Irish cuisine, despite the rise of globalization and increased availability of foreign foods (Mac Con Iomaire, 2018). Potatoes, bread, dairy products, cereals, meat, and vegetables remain the staples of Irish cuisine (Mac Con Iomaire, 2011). While some of the food pairings selected in this study may be familiar in some regions of the globe (such as the Caribbean and the United States), there would be less exposure to such pairings in Irish society. The methods for this study were approved by the Research Ethics Committee at Technological University Dublin (TU Dublin).

\section{Phase 1: attitudes and reactions toward novel food pairings, a qualitative analysis}

Three focus groups were conducted to explore the participants' attitudes and reactions toward the novel pairing of foods. Previous research has demonstrated that groups, consisting of three or more people, are beneficial for generating an open dialogue that is comfortable for the participants and meaningfully varied (Carlsen \& Glenton, 2011). The researchers stopped after three focus groups because data saturation was achieved as no new information was being revealed (Bowen, 2008). During the focus groups, the participants were presented with eight novel food pairings as odor stimuli. The odors of the stimuli were assessed as opposed to having the participants consume the stimuli as many of the odor stimuli are considered as allergens. Additionally, many of the stimuli have complex and strong flavors and tastes (blue cheese, soy sauce, etc.), as such, assessment of all the stimuli through consumption in a single session may cause palate fatigue for participants. In this sense, while the odor of stimuli did represent the actual flavor of the ingredients, it did exemplify the expected flavor of the stimuli. 


\section{Description of focus groups and topics}

The focus groups consisted of eight, seven, and seven participants, respectively $(n=22)$. All participants resided in Ireland; an overview of the sample is provided in Table 1 . Inclusion criteria for this study were age ( $>18$ years), staff or graduate students enrolled in the School of Culinary Arts and Food Technology, and the School of Food Science and Environmental Health, TU Dublin, Ireland. The participants were divided across the three focus group sessions based on their availability and convenience. The focus groups were conducted according to standard procedures (Krueger \& Casey, 2009). The setting was a conference room, and audio recordings of the focus groups were conducted on personal computers using sound recording and editing software (Audacity 1.3, The Audacity Development Team). Each focus group lasted between 90 and 120 min and was guided by a moderator. Participants were provided with information regarding the general rules for performing each session and were familiarized with the subject matter of the "novel food pairing" via a 30 -min presentation by the moderator.

A topic guide was developed before the fieldwork and consisted of open discussions on (1) the concept of pairing foods based on shared flavor compounds, (2) familiar and traditional food pairings, and (3) novel/unfamiliar food pairings. Visual cues, in the form of photographs of culturally accepted traditional (Irish culture) and non-traditional food pairings, were used throughout the focus group sessions to relate to the food pairings being discussed. A diagram of the initial 40 novel foods paired with banana generated by the software was presented to the participants for discussion regarding the familiarity and opinions of these pairings. Participants were encouraged to share any familiar and unfamiliar food pairings that they have experienced that were not included in the visual cues.

Table 1. Overview of participant for focus groups $(n=22)$.

\begin{tabular}{lc}
\hline Sample & $n$ \\
\hline Gender & \\
Male & 7 \\
Female & 15 \\
Age (years) & \\
$18-24$ & 18 \\
$25-34$ & 6 \\
Nationality & \\
Irish & 18 \\
Polish & 3 \\
French & 1 \\
Participant categorization & \\
Food science students & 14 \\
Culinary arts students & 5 \\
Staff & 2 \\
Graduate students & 1 \\
\hline
\end{tabular}




\section{Odor stimuli (aroma pairings)}

Following the initial discussion during the focus groups, participants were presented with the novel food pairings as odor stimuli for assessment, and open discussions regarding the pairings were conducted. The selection of the odor stimuli was performed through preliminary research. This involved the pairing of foods (animal and non-animal origin) with fresh banana as the primary-paired ingredient (Cavendish, origin Costa Rica) to create non-traditional and unfamiliar pairings in the context of the research setting, Ireland. Banana was selected as the primary ingredient in each pairing as it is widely considered a pleasant tasting fruit and is one of the highest consumed fruits throughout the world (Jordán, Tandon, Shaw, \& Goodner, 2001; Mayr, Märk, Lindinger, Brevard, \& Yeretzian, 2003). The pairings for the stimuli were generated using the online software package Foodpairing.com (Sense for Taste, Bruges, Belgium). The software uses an algorithmic-assisted food pairing program based on the 'Food Pairing Theory.' The underlying hypothesis of this theory is that foods may combine well when they share some common characteristic (dominant) aromatic volatiles compounds (Kort, Nijssen, van Ingen-visscher, \& Donders, 2010; Perkel, 2018). This approach to pairing foods based on commonly shared aromatic compounds has been used in the past to produce successful, yet unusual, culinary combinations such as white chocolate and caviar, salmon and licorice, and banana and parsley (de Klepper, 2011; Spence, Wang, \& Youssef, 2017). Despite current empirical research lacking support for the underlying hypothesis of this theory (Ahn, Ahnert, Bagrow, \& Barabasi, 2011; Kort et al., 2010; Traynor, Burke, O’Sullivan, Hannon, \& BarryRyan, 2013), the approach is considered to be a useful tool to support culinary creativity and innovation (Spence et al., 2017).

The food pairing software suggested over 40 foods to be paired with banana. In order to reduce this number to a more manageable quantity (for experimental purposes), factors such as availability and food expense were considered. In addition, two professional chefs, two culinary instructors, and a culinary historian were consulted regarding the novelty of the selected pairings in Irish cuisine. Careful consideration was taken to include foods of both animal and non-animal origin. The selected foods used as odor stimuli were bacon, basmati rice, extra virgin olive oil, blue cheese, Gruyère cheese, mackerel, soy sauce, and whole grain mustard.

Bananas were purchased at stage 5, yellow with green tips, based on a 7-point banana peel color scale (Meng, David, \& James, 1997). They were allowed to ripen to stage 6 , entirely yellow, in-room temperature conditions. All other food ingredients were purchased and cooked on the day of holding the focus groups. The quantity of each food item to be used was determined through preliminary sensory analysis $(\mathrm{n}=5)$, where a perceived aroma balance (aroma intensity) was achieved to ensure that no single aroma dominated the aroma profile. All food ingredients were purchased and cooked on the day of holding the focus groups. 
The aroma of the odor stimuli was assessed in a sensory laboratory under guidelines and conditions according to ISO 8589:2010 (International Organisation for Standardization [ISO], 2010). Samples were prepared according to Raz et al. (2008); at least $30 \mathrm{~min}$ prior to testing and held at ambient temperature $\left(18-20^{\circ} \mathrm{C}\right)$ to allow for vapor pressure to reach equilibrium. The food items were placed in opaque plastic containers with opaque lids so that respondents could smell them without seeing. Five minutes before the presentation of the food, small holes were made in the lids with a needle, through which the aromas were released. The samples of banana were presented to the participants, which they retained for the remainder of the focus group session. The eight samples to be paired with the banana were presented in a monadic sequential (one sample at a time) and randomized order. Participants were instructed to assess the banana sample and the paired sample together at the same time; hold the banana sample in one hand and the pairing sample in the other hand, hold the samples just below the nose and take three short sniffs with the mouth closed. Participants were allowed to assess each sample pairings for as long as needed and were able to revisit sample pairings if required. The odor stimuli were presented blindly to each participant to allow for assessment of the aroma of each pairing before the components of the pairing combinations were revealed. A discussion of the odor stimuli directly followed the assessment of the odor stimuli. The participants discussed their familiarity (or lack of familiarity) with the stimuli, hedonic reactions to the odor of the stimuli, the expected flavors of the pairings, the overall opinion of the pairings, and in what form of food they would like to see the food pairings presented.

\section{Qualitative analysis of focus group data}

The focus group recordings were transcribed verbatim and typed into a Word document. In the second round of transcription, the researchers returned to the audio recordings and noted the tone and emotional expression (if any was clearly obvious) within the transcription document. Once this process was complete, the three researchers independently analyzed the audio and transcriptions. The goal was to conduct a thematic analysis, determining what themes emerged from the data. This analysis would help to understand the underlying motivations for the participants' reactions to traditional and novel food pairings. In addition to themes, the researchers noted the valence (positive, neutral, or negative) of each segment of datum. In this way, themes were matched with valences throughout the analysis. Once the text was independently coded, the three separate analyses were triangulated, and any discrepancies were discussed and reconciled through discussion until an agreement was reached on themes, valences, and tones (Braun \& Clarke, 2006; Creswell, 2007). 


\section{Phase 2: hedonic assessment of novel food products, quantitative analysis}

A consensus among the focus group participants regarding the preferred food pairings was banana and bacon (animal sample), and banana and rice (nonanimal sample). The focus group participants also agreed that an ice cream product would be an ideal product to present the novel food pairings to a consumer panel. The findings from the focus group were used to develop two novel ice-cream products, one with animal-based flavorings and one non-animal based flavorings, for the second phase of the study (quantitative study). The ice cream samples were developed through extensive preliminary testing and sensory evaluation $(\mathrm{n}=34)$ to optimize the flavor ingredient concentrations. For sample preparation and flavor profile optimization findings, see Traynor (2013). Furthermore, to investigate the impact of culinary education on overall liking of these novel food products, a general cohort (control) and a culinary-educated cohort were recruited. Inclusion criteria for this study were age (>18 years), enrollment in a culinary arts degree (not enrolled for control cohort, and enrolled for culinary-educated cohort), and consumption of ice cream products (must consume ice cream products).

\section{Sensory evaluation of ice creams}

The control cohort consisted of 76 panelists (30 males and 46 females; between 18 and 34 years). Panelists were recruited from students of non-culinary degrees at the School of Food Science and Environmental Health at TU Dublin, Ireland. The culinary-educated cohort consisted of 76 panelists $(n=76 ; 22$ males and 54 females; between 18 and 34) and were recruited from culinary classes at the School of Culinary Arts and Food Technology in TU Dublin. Prior to testing, panelists were informed that they would be evaluating novel ice creams. A list of ice cream ingredients was provided on the consent form to allow for the withdrawal of participation due to potential religious, food preference, or allergy reasons. Panelists were asked to evaluate the overall liking and their willingness to purchase the two ice cream samples.

Sensory evaluation took place in a sensory laboratory under guidelines and conditions according to ISO 8589:2010 (ISO, 2010). Each panelist worked in a single booth under defined conditions of $22^{\circ} \mathrm{C}$ and white light. Approximately $30 \mathrm{~g}$ of each ice cream was placed in sample containers coded with three-digit random numbers. Samples were presented in a monadic sequential (one sample at a time) and randomized order to the panelist's and were served at between $-11^{\circ} \mathrm{C}$ and $-13^{\circ} \mathrm{C}$ (Aime, Arntfield, Malcolmson, \& Ryland, 2001). Panelists were instructed to evaluate the overall liking of the ice creams on a 9-point Likert scale, where 9 = "like extremely," 5 = "neither like nor dislike" and 1 = "dislike extremely," and to consume mineral water to rinse their mouths between evaluating each sample. Panelists were asked to indicate their willingness to purchase the samples (willing to purchase or not willing to purchase). In addition, qualitative 
data were recorded; panelists were encouraged to write comments regarding their overall opinion of the ice creams. These comments were analyzed for their valence; positive, negative, or neutral.

\section{Statistical analysis of sensory evaluation data}

Tests for normality (Shapiro-Wilk test) showed that the data did not follow a normal distribution. For this reason, a Mann-Whitney $U$ test was used to compare the overall liking results of the control cohort to those of the culinary-educated cohort for each ice cream sample. Pearson's chi-square tests were conducted on the willingness to purchase data and the sensory evaluation comments data to test for associations between cohort type willingness to purchase, between cohort type and comment valence (positive, neutral, and negative). The significance level for the statistical tests was $\mathrm{p} \leq 0.05$, and the analysis was performed using SPSS program for Windows (version 19.0, SPSS Inc., Chicago, IL, USA).

\section{Results and discussion}

\section{Phase 1 focus groups}

The preceding observations about food pairings by the focus group members precipitated a further examination of the qualitative data, specifically looking for the motivations of the acceptance or rejection of the pairings (Table 2). These themes appeared to align with the three fundamental motivational dimensions of Rozin and Fallon's (1987) taxonomy for reactions to food; Sensory-Affective reactions, Anticipated Consequences, and Ideational (disgust and cultural appropriateness).

\section{Sensory-affective reactions}

A primary emergent theme from the analysis was Sensory-Affective reactions. Both positive and negative valences of this motivational dimension were identified and varied depending on the stimuli and discussion point (familiar (traditional) or unfamiliar (novel) food pairings). The first subtheme was that of familiar traditional pairings, in which participants appeared to unanimously react positively toward the visual cues presented, "I have tried the venison and chocolate, it was good."

\section{Unfamiliar pairings}

A second subtheme was the mixed Sensory-Affective reactions amongst participants of all three focus groups when discussing unfamiliar novel food pairings. During the discussion of novel flavor pairings experienced in 
Table 2. Summary of emergent themes and subthemes with valences from analysis of focus groups $(n=22)$.

\begin{tabular}{|c|c|c|}
\hline Themes & Subthemes & Valence \\
\hline \multirow[t]{3}{*}{ Sensory-Affective } & $\begin{array}{l}\text { a. Familiar traditional pairings created using complementary ingredients to } \\
\text { produce harmonious combined flavor profiles. }\end{array}$ & + \\
\hline & $\begin{array}{l}\text { b. Mixed reactions (positive and negative valences) toward the sensory } \\
\text { characteristics of unfamiliar novel food pairings. }\end{array}$ & $+/-$ \\
\hline & c. Adding a level of familiarity/resemblance to a novel food pairing. & + \\
\hline \multirow[t]{5}{*}{ Ideational } & a. Disgust of familiar and unfamiliar animal-based products (organ meat). & - \\
\hline & b. Generational differences regarding disgust of traditional meat products. & - \\
\hline & c. Disgust based on knowledge of origin and nature of food item. & - \\
\hline & $\begin{array}{l}\text { d. Sensory characteristics overriding mental thoughts of foods evoking } \\
\text { disgust. }\end{array}$ & \\
\hline & e. Cultural appropriateness of foods. & \\
\hline $\begin{array}{l}\text { Anticipated } \\
\text { consequences }\end{array}$ & a. Safe consumption of insects in certain cultures (entomophagy) & + \\
\hline
\end{tabular}

their personal life, several participants expressed negative reactions toward the sensory characteristics of some of the unfamiliar novel pairings shared by fellow focus group participants. This manifested through apparent surprise and discomfort amongst participants. An example was the discussion of the pairing of cauliflower with curry. Some participants had consumed this pairing at a restaurant and explained that the taste "was disgusting" and "just wrong." In contrast, another participant indicated that they had enjoyed that particular food pairing. This suggests that while food preferences and familiarity of food are frequently transferred within a culture, subculture, and family (Tuorila, 1996), in certain instances, hedonic responses can be more of an individual experience.

\section{Resembling familiar flavors}

A third subtheme was creating a novel pairing that resembles a familiar flavor. This 'hidden familiarity' was more apparent in the reactions towards the pairing of 
rice and banana. Many participants in all of the three focus groups noted that this particular pairing produce an aroma profile that resembled the aroma of familiar food items, "I got a smell of popcorn from it [rice and banana pairing]," "I think it is biscuits," "maybe crackers," "I thought it was [potato] crisps or something like that," and "I got a smell of peanuts when I combined the banana with rice." Thus, it can be inferred that creating an unfamiliar food pairing that results in a certain degree of resemblance to the flavor profile of a familiar food may positively impact the hedonic reaction to that pairing. A known method of decreasing negative reactions to novel foods requires a sufficient level of familiarity (Rozin \& Rozin, 1981). Other studies have reported that pairing familiar foods that were liked with unfamiliar foods increased their liking (Anzman-Frasca, Savage, Marini, Fisher, \& Birch, 2012; Johnston et al., 2011; Pliner \& Stallberg-White, 2000).

\section{Ideational (origins/nature)}

\section{Disgust}

Ideational (origins/nature) motivation was another common theme that emerged from the analysis. More specifically, disgust and cultural appropriateness were two main emergent subthemes of this motivational dimension. For disgust, a negative valence toward familiar and unfamiliar animal-based ingredients (meat, fish, cheese, insect, etc.) was a common reaction that transpired during all of the focus groups. Many participants explained that while older generations frequently consumed offal without hesitation or disgust, younger generations demonstrate more disgust toward these products based on the knowledge of the origin of the meat (organ), "I would not eat much offal. Liver or kidney or anything like that."

\section{Food origins}

The disgust subtheme was evident during the assessment and discussion of the odor stimuli. Most participants displayed reactions of disgust toward pairings containing animal-based ingredients (mackerel, blue cheese, and bacon). While many participants appeared to initially liked the aromas of some of the odor stimuli during the blind assessment, some of the participant's reactions changed to disgust once the ingredients were revealed by the researchers. This demonstrated disgust based on the nature and origin of the food item itself rather than the sensory characteristics.

\section{Sensory overriding}

Another interesting finding was that pleasing organoleptic characteristics (flavor, taste, etc.) of a food item may override the certain levels of disgust toward particular foods and pairings. When discussing a novel food pairing mentioned by one the focus participants ("pork sausage with hummus"), it was explained that while the thought of the combination was not appealing or appetizing, the actual 
flavor of the combination was quite pleasing, "it should not taste good, but it actually does." Previous researchers have found that ideational motivations are important factors in the rejection of unfamiliar foods (Ammann et al., 2018a, 2018b; Hartmann \& Siegrist, 2018), especially in the negative response to unfamiliar animal-based foods (Martins et al., 1997; Martins \& Pliner, 2005, 2006; Pliner, 1994; Tan et al., 2015).

\section{Cultural appropriateness}

A final subtheme under Ideational motivations was cultural appropriateness. It appeared that participants considered the acceptance of the pairing of foods as learned from experience and/or exposure mainly through cultural and environmental influences as opposed to being innate (Bodenheimer, 1951; Rozin \& Vollmecke, 1986; Tan et al., 2015).

Many participants indicated that entomophagy was considered notably more appropriate, familiar, and widely consumed across non-Western cultures than in Western cultures where it can be considered repulsive, "it is one of the specialties [in Thailand], all the Thai people will go eat that [fried bugs]." This statement was met by uncomfortable laughter among the panelists, followed by a compliment from another participant saying, "fair play to you, I don't know if I could." This sentiment aligns with previous literature that examined the acceptance of food through the lens of cultural appropriateness and concluded that culture is a significant factor in acceptance or rejection of a food (Tan et al., 2015). Generally speaking, food choices are culturally determined and can vary significantly across cultural groups, this is very much the case with entomophagy (Deroy, Reade, \& Spence, 2015).

\section{Anticipated consequences}

The emergent theme of Anticipated Consequences was less evident compared to the themes of Sensory-Affective and Ideational motivations in the analysis. Anticipated Consequences are often associated with whether the item is wholesome or harmful (Rozin \& Fallon, 1987). The positive pole of this dimension was evident when discussing entomophagy in Thai culture. One participant stated that she had tried the fried locusts, which were served as snacks in Thailand. It was explained that "all the Thai people will eat that [fried insects, therefore] it cannot be poisonous, so it's okay to eat."

\section{Phase 2: sensory evaluation of novel products}

H1: A culinary-educated cohort will like a novel animal ice cream product significantly more than a control cohort 
A Mann-Whitney $U$ test was carried out to assess the difference in the overall liking of the novel ice cream samples between the two different cohorts, a control cohort and a culinary-educated cohort. The results showed that the animal sample, banana, and bacon ice cream was liked significantly more by the culinary-educated cohort $(\mathrm{Mdn}=6.90)$ than by the control cohort $(\mathrm{Mdn}=5.40), U=1612.00, p=.000$ (Table 3). Thus, for this novel food product, the null hypothesis was rejected. The qualitative data collected during the hedonic testing supported these findings (Figure 1). The chi-square analysis of this data revealed a significant relationship between cohort type and type valence of comment for the animal-based sample $\left(X^{2}(2, \mathrm{n}=123)=13.333, p=.001\right)$. The comments regarding the animal sample were mainly negative amongst the control cohort (52.6\%). Moreover, the positive comments only account for $31.6 \%$ of comments from the control cohort regarding the animal-based sample. Many panelists reported that they "didn't like the unusual taste," "the thought of the bacon taste would throw me off [consuming this]," and found it "too salty for an ice cream."

In contrast, the feedback from the culinary-educated cohort was predominantly positive (63.6\%). Panelists reported that they "liked the sweet and salty taste combination" and that the flavor was "very interesting." Furthermore, chi-squared analysis of the willingness to purchase data revealed that there was a significant association between cohort type and willingness to purchase (Figure 2$)\left(X^{2}(1, \mathrm{n}=152)=17.544, p=.000\right)$. Only $46.0 \%$ of the control cohort reported a willingness to purchase in comparison to $79.3 \%$ of the culinary-educated panelists (Figure 2).

H2: A culinary-education cohort will like a novel non-animal ice cream product significantly more than a control cohort

For the non-animal sample, banana, and rice ice cream, no significant differences were found between overall liking scores between the control cohort $(\operatorname{Mdn}=7.38)$ and culinary-educated cohort $(\operatorname{Mdn}=6.90)$,

Table 3. Median overall liking scores with interquartile range for the hedonic assessment (overall liking) of an animal ice cream and a non-animal ice cream by a control cohort $(n=76)$ and a culinary-educated cohort $(n=76)$.

\begin{tabular}{llcccc}
\hline \multirow{2}{*}{ Sample } & \multicolumn{2}{c}{ Overall liking median (IQR) } & & \multicolumn{2}{c}{ Mann-Whitney U } \\
\cline { 2 - 3 } $\begin{array}{l}\text { Animal based ice cream: } \\
\text { banana and bacon }\end{array}$ & $5.4(4.0)$ & $6.9(2.1)$ & & 1612.0 & -value \\
$\begin{array}{l}\text { Non-animal based ice cream: } \\
\text { banana and rice }\end{array}$ & $7.4(1.5)$ & $6.9(1.1)$ & & 2432.5 & .000 \\
\hline
\end{tabular}

Overall liking values are median 9-point Likert scale.

IQR: interquartile range. 


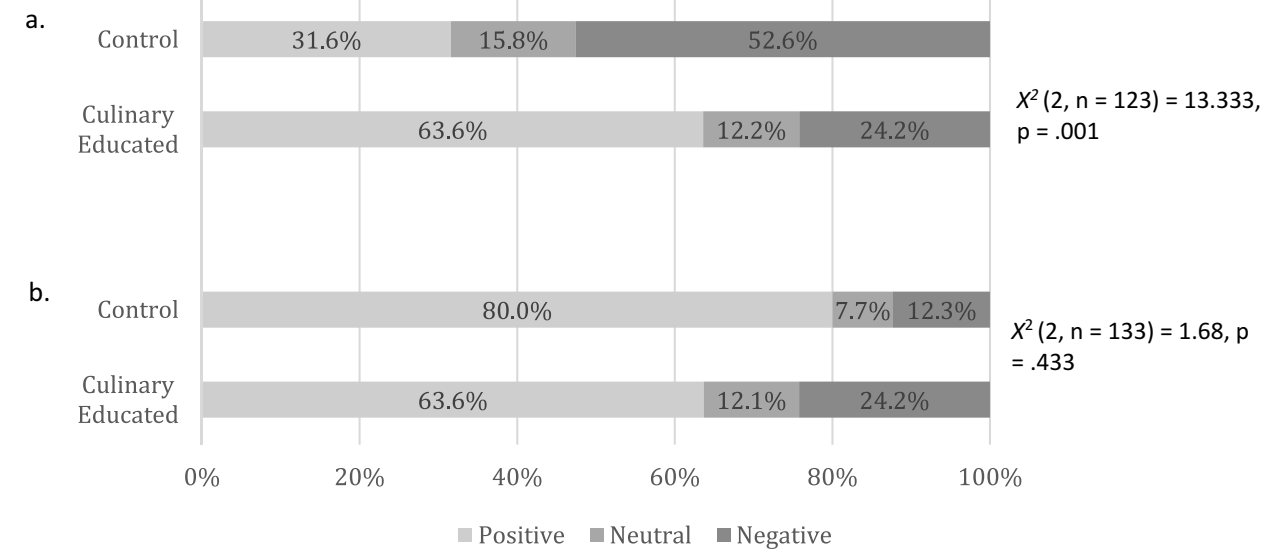

A - Animal ice cream sample

B - Non-animal ice cream sample

Figure 1. Percentage of valence (positive, neutral, and negative) for sensory evaluation panelist comments for (a) an animal ice cream product and (b) a non-animal ice cream product by a control cohort (57 responses) and a culinary-educated cohort (66 responses).

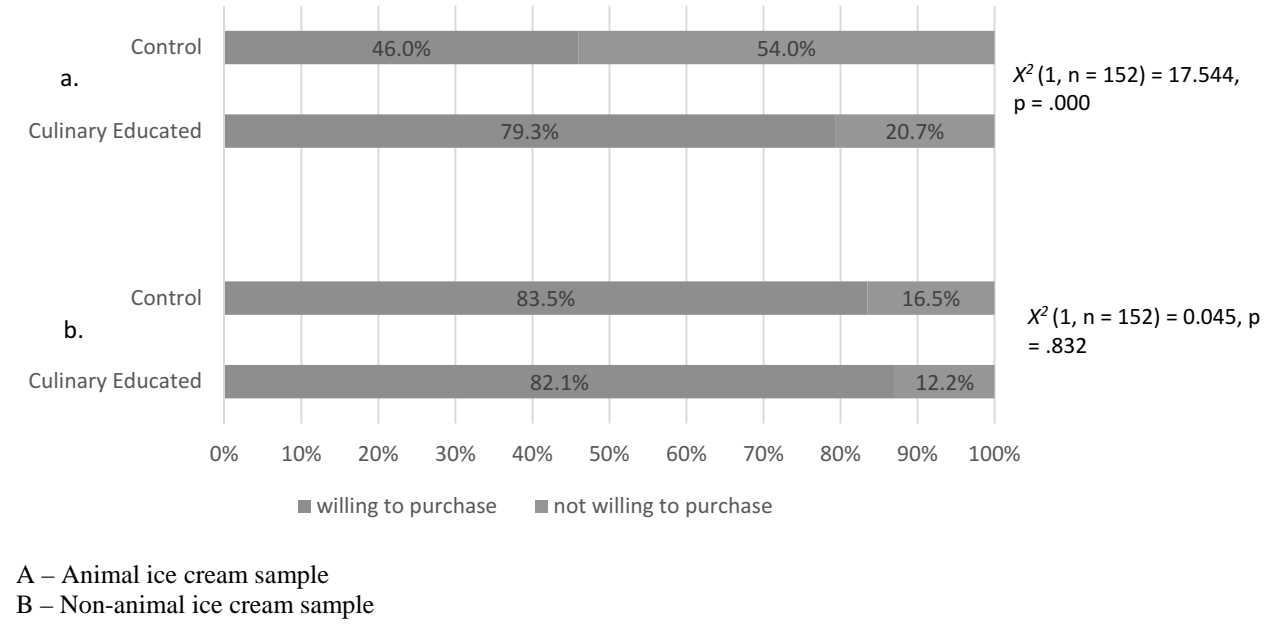

Figure 2. Percentage of response for willingness to purchase for (a) an animal product and (b) a non-animal product by a control cohort and a culinary-educated cohort.

$U=2435.5, p=.194$ (Table 3). Therefore, the null hypothesis could not be rejected. In addition, the chi-square analysis revealed no significant association between cohort type and type valence of comment for the non-animal sample $\left(X^{2}(2, \mathrm{n}=133)=2.68, p=.433\right)$. The comments regarding the nonanimal ice cream were mainly positive from both cohorts, with $80.0 \%$ for the control cohort and $75.0 \%$ for the culinary-educated cohort (Figure 1). Many assessors in both panels stated that the ice cream had "pleasant flavors" that "[the taste and flavor] work well together and are not overpowering." 
Concerning willingness to purchase, a comparable $83.5 \%$ of the control cohort and $82.1 \%$ of the culinaryeducated cohort indicated a willingness to purchase (Figure 2). Moreover, there was no significant association between cohort type and willingness to try the non-animal sample $\left(X^{2} \quad(1\right.$, $\mathrm{n}=152)=0.045, p=.832$ ). The comments from the panelists imply that non-animal ice cream was a more naturally sweeter tasting pairing in comparison to the more complex and contrasting tastes (sweet and salty) and flavor of the banana and bacon pairing (animal sample). This may be a fact in it being perceived as more acceptable and harmonious. Sweeter tastes and flavors have been shown to evoke fewer negative reactions and tendencies when tasting unfamiliar foods (King et al., 2008).

\section{General discussion}

In the current study, it was evident that when discussing the novel pairing of the foods and flavors, exposure to unfamiliar foods impacted attitudes and behaviors in both phases of the study. For the first phase, it was clear that the participant's attitude toward traditional and non-traditional food pairings was consistent with the three fundamental motivational dimensions of Rozin and Fallon's (1987) taxonomy for reactions to food; Sensory-Affective reactions, Anticipated Consequences, and Ideational (disgust and cultural appropriateness). For the second phase of the study, the reactions to the novel food products appeared to be multifaceted and quite complex in nature. While the influence of culinary education may provide some explanation for the differences in hedonic responses, the findings from the focus groups also provide further insight regarding the potential underlying motivations resulting in the specific reactions amongst the two cohorts.

A post hoc analysis of the open-ended responses from panelists in the second phase of the study, using the a priori categorization determined from the first phase, was conducted. The control group demonstrated a negative reaction to the animal ice-cream across the two motivational dimensions Ideational and Sensory-Affective, while the culinary-educated group demonstrated a positive reaction, Sensory-Affective appeared to be the sole underlying motivational dimension. With the non-animal ice cream, the control group demonstrated positive reactions and were motivated by Sensory-Affective and Ideational factors, whereas the culinary-educated group was only positively motivated by Ideational factors.

Regarding the noteworthy differences in the hedonic responses toward the animal product between the different cohorts suggests that both positive and negative poles of the Sensory-Affective dimension were underlying motivations for the reactions toward the animal sample. For the culinary-educated cohort, positive Sensory-Affective reactions ("good tastes") toward this seemingly more complex tasting sample predominated the overall acceptance. In 
contrast, feedback regarding the "unusual taste" of the sample would suggest that the negative pole of this dimension, distaste, was the main motivational factor in the rejection by the control panel.

Furthermore, it can also be inferred that there was also a certain degree of Ideational motivation in the form of inappropriateness resulting in the rejection of this animal sample by the control cohort. The knowledge of the nature of the main ingredient which was of animal origin (bacon), denoted by the negative feedback from panelists centered around "the thought of the bacon taste," may have resulted in this sample being considered inappropriate, and therefore rejected (Martins et al., 1997). As was the case with the non-animal sample, the main flavor ingredients, banana and bacon, are culturally appropriate and socially accepted edible foods in Ireland. However, the combination of both ingredients, in the form of a sweet dessert, seemed to have resulted in an adverse reaction (rejection) in the control cohort, possibly motivated by a feeling of cultural inappropriateness toward this sample.

Another potential explanation may be due to a disconfirmation concerning the sensory expectation. A study by Yeomans, Chambers, Blumenthal, and Blake (2008) found that the label given during a sensory test to a highly novel ice cream played a significant role in generating hedonic responses to the food. They found marked differences in the expected and actual liking of a smoked-salmon ice cream depending on the associated label. A strong negative affective response (dislike and rejection) and an enhancement of the unexpected sensory qualities (salty fish) was found when the product was labeled as an "ice cream" in contrast to when it was labeled as "frozen savory mousse." In the current study, panelists were informed that they would be evaluating ice cream products, thus had a prior expectation and anticipation of a sweet product. However, the animal-based product was found to be more savory with a noted "bacon and salty taste." While the phenomena of hedonic expectation may have played a role in the hedonic responses, the expected liking of the products was not measured and therefore is a limitation of the study.

In relation to the non-animal sample, it can be extrapolated that the mainly positive hedonic reaction of this sample, denoted by the relatively high overall liking scores, a higher degree of willingness to purchase, and mostly positive comments, was motivated by positive poles of the AffectiveSensory and Ideational dimensions. As was prevalent in the focus groups, environmental influences such as social and cultural appropriateness (Ideational dimension) appeared to have impacted how the control cohort evaluated the unfamiliar products.

Individually, banana and rice are common ingredients used separately in traditional ways, in Ireland, to produce dessert products with specific sensory properties that are considered traditional local products in local context and 
culture (Stolzenbach, Bredie, \& Byrne, 2013). While combining these ingredients in the novel ice cream produced an unfamiliar product, it appeared that the flavor profile resembled a more familiar and traditional product to the panelists than the animal product (banana and bacon), and thus may be considered culturally appropriate and produced an expected pleasant sweet and fruity flavor. In fact, in the focus groups, numerous participants stated that the ingredient combination of banana and rice is more favorable for a dessert product and that a "rice pudding" dessert would be a suitable product for pairing them.

Exposure, through culinary education, may explain these differences in the potential underlying motivations between the reactions of the different cohorts for the assessment of the animal sample. In this study, the subjects of the culinary-educated cohort were enrolled in university culinary classes, and thereby frequently discussed gastronomy and the concept of pairing foods and flavors, were exposed to diverse cuisines, practice, and mastered professional culinary techniques. Hence, similar to the findings of Reverdy et al. (2010), the culinary centered education most likely increased this cohort's culinary vocabulary and their interest (involvement) with food and flavors. Furthermore, the inherent nature of the culinary laboratory classes in which this cohort would participate may increase the likelihood of exposure to moderately novel, arousing, and complex stimuli (food ingredients and culinary pairings) regularly and for a prolonged period (from 2 to 4 years). Thus, the nature of the educational program most likely reduced the probability of negative responses and increased the positive responses to the novel stimuli in this study. Complexity is known to be a substantial factor in altering the arousal levels (Lévy, MacRae, \& Köster, 2006). Thus, the more complex food pairing in terms of flavor of banana and bacon ice cream ("salty and meaty taste") in comparison to the banana and rice ice cream appeared to serve as a more arousing food product for the culinary-educated cohort (Reverdy et al., 2004) and therefore evoked positive Sensory-Affective reactions. The control cohort, as a whole, seemingly had considerably less exposure to novel and complex food and flavor pairings in comparison to the culinary-educated cohort.

Considering the motivational dimensions underlying acceptance and rejection of foods are bipolar, the induction of the positive poles of these dimensions may encourage acceptance of novel foods (Martins et al., 1997; Martins \& Pliner, 2006). Therefore, exposure to foods with more complex sensory properties (flavor and taste) through culinary education, as a learning mechanism, may increase the likelihood of the positive poles of the motivational dimensions influencing the reactions (acceptance) toward novel animal-based foods in adults. These findings can help direct foodservice and food manufacturing professionals (research chefs) in the experimentation and development of new innovative culinary creations by 
shedding light on their target markets' underlying motivations for the acceptance and rejection of such foods and flavors. For instance, if the target market is a cohort with minimal knowledge of and exposure to novel and complex foods, developing a product with a less pronounced and complex flavor profile that resembles more familiar food flavor may produce more promising outcomes in terms of acceptance.

The current study was limited by the use of a narrowly defined sample cohort, the participants in this study were not representative of all university adults. Expanding the study across different cultures and as a longitudinal study may provide more generalizable observations and insight into the impact of culinary education on attitudes and behaviors toward novel flavors.

\section{Conclusions}

In this exploratory study, the mixed-methodology approach allowed for an expanded understanding of the attitudes and behaviors toward unfamiliar animal and non-animal food pairings. Furthermore, this study explored the effect that culinary education may have on reactions to novel food pairings. The Sensory-Affective and Ideational dimensions appeared to be key motivational factors for the reactions to unfamiliar food pairings. More specifically, the environmental influences of social and cultural appropriateness seemed to have impacted how the control cohort evaluated the animal sample. The findings in this study support that of other researchers, where differences were found between reactions to animal and non-animal foods.

An important finding was that participants exposed to culinary education had a greater overall liking for the novel animal food pairing compared to the control group, despite that pairings were developed through focus groups with both culinary educated and control subjects. Positive Sensory-Affective reactions seemed to be motivational factors when explaining these findings. Conversely, both negative Sensory-Affective and Ideational (inappropriateness) appeared to be motivational factors in terms of assessment by a cohort without culinary education. As one would expect, the acceptance of the intrinsic sensory properties of novel foods is an important factor to consider when developing food products. However, the extrinsic motivations, such as concerns regarding the origin of foods and emotional reactions, can also have significant impacts on the acceptance of novel foods.

\section{References}

Ahn, Y. Y., Ahnert, S. E., Bagrow, J. P., \& Barabasi, A. L. (2011). The flavor network and the principles of food pairing. Scientific Reports, 1, 196. doi:10.1038/srep00196 
Aime, D., Arntfield, S., Malcolmson, L., \& Ryland, D. (2001). Textural analysis of fat reduced vanilla ice cream products. Food Research International, 34(2-3), 237-246. doi:10.1016/ S0963-9969(00)00160-5

Ammann, J., Hartmann, C., \& Siegrist, M. (2018a). Development and validation of the food disgust picture scale. Appetite, 121(1), 367-379. doi:10.1016/j.appet.2018.02.020

Ammann, J., Hartmann, C., \& Siegrist, M. (2018b). Does food disgust sensitivity influence eating behavior? Experimental validation of the food disgust scale. Food Quality and Preference, 68, 411-414. doi:10.1016/j.foodqual.2017.12.013

Anzman-Frasca, S., Savage, J., Marini, M. E., Fisher, J. O., \& Birch, L. L. (2012). Repeated exposure and associative conditioning promote preschool children's liking of vegetables. Appetite, 58(2), 543-553. doi:10.1016/j.appet.2011.11.012

Asperin, A., WooMi, P., \& Wolfe, K. (2011, July). Exploring food neophobia and perceptions of ethnic foods the case of Chinese and Thai cuisines. Proceedings of the International CHRIE Conference, Denver, CO, ScholarWorks@UMass.

Backstrom, A., Pirttila-Backman, A. M., \& Tuorila, H. (2003). Dimensions of novelty: A social representation approach to new foods. Appetite, 40(3), 299-307. doi:10.1016/S01956663(03)00005-9

Backstrom, A., Pirttila-Backman, A. M., \& Tuorila, H. (2004). Willingness to try new foods as predicted by social representations and attitude and trait scales. Appetite, 43(1), 75-83. doi:10.1016/j.appet.2004.03.004

Barrena, R., \& Sánchez, M. (2012). Neophobia, personal consumer values and novel food acceptance. Food Quality and Preference, 27(1), 72-84. doi:10.1016/j. foodqual.2012.06.007

Bodenheimer, F. S. (1951). Insects as human food: A chapter of the ecology of man. Dordrecht, Netherlands: Springer.

Bowen, G. A. (2008). Naturalistic inquiry and the saturation concept: A research note. Qualitative Research, 8(1), 137-152. doi:10.1177/1468794107085301

Braun, V., \& Clarke, V. (2006). Using thematic analysis in psychology. Qualitative Research in Psychology, 3, 77-101. doi:10.1191/1478088706qp063oa

Carlsen, B., \& Glenton, C. (2011). What about N? A methodological study of sample-size reporting in focus group studies. BMC Medical Research Methodology, 11, 26. doi:10.1186/ 1471-2288-11-26

Chung, L., Chung, S. J., Kim, J. Y., Kim, K. O., O’Mahony, M., Vickers, Z., Cha, S.-M., Ishii, R., Baures, K., \& Kim, H.-R. (2012). Comparing the liking for Korean style salad dressings and beverages between US and Korean consumers: Effects of sensory and non-sensory factors. Food Quality and Preference, 26(1), 105-118. doi:10.1016/j.foodqual.2012.03.011

Creswell, J. (2007). Qualitative inquiry and research design: Choosing among five approaches (2nded.). Thousand Oaks, CA: Sage.

Creswell, J. W., Plano Clark, V. L., Gutmann, M., \& Hanson, W. (2003). Advanced mixed methods research designs. In A. Tashakkori \& C. Teddlie (Eds.), Handbook of mixed methods in social and behavioral research (pp. 209-240). Thousand Oaks, CA: Sage.

de Klepper, M. (2011). Food pairing theory: A European fad. Gastronomica Journal of Food Culture, 11(4), 55-58.

Deroy, O., Reade, B., \& Spence, C. (2015). The insectivore's dilemma and how to take the West out of it. Food Quality and Preference, 44, 44-55. doi:10.1016/j. foodqual.2015.02.007

Elkins, A., \& Zickgraf, H. F. (2018). Picky eating and food neophobia: Resemblance and agreement in parent/young adult dyads. Appetite, 126, 36-42. doi:10.1016/j. appet.2018.02.021 
Gibson, E. L., \& Brunstrom, J. M. (2007). Learned influences on appetite, food choice and intake: Evidence in human beings, in progress in brain research. In S. J. Cooper \& T. C. Kirkham (Eds.), Appetite and body weight: Integrative systems and the development of anti-obesity drugs (1 ed., pp. 271-300). London, UK: Academic Press.

Greene, J. C., Caracelli, V. J., \& Graham, W. F. (1989). Toward a conceptual framework for mixed-method evaluation designs. Educational Evaluation and Policy Analysis, 11(3), 255-274. doi:10.3102/01623737011003255

Hartmann, C., \& Siegrist, M. (2018). Development and validation of the food disgust scale. Food Quality and Preference, 63, 38-50. doi:10.1016/j.foodqual.2017.07.013

Henriques, A., King, S., \& Meiselman., H. (2009). Consumer segmentation based on food neophobia and its application to product development. Food Quality and Preference, 20(2), 83-91. doi:10.1016/j.foodqual.2008.01.003

Hoppu, U., Prinz, M., Ojansivu, P., Laaksonen, O., \& Sandell, M. A. (2015). Impact of sensory-based food education in kindergarten on willingness to eat vegetables and berries. Food and Nutrition Research, 59(1), 1-8. doi:10.3402/fnr.v59.28795

International Standards Organisation. (2010). ISO 8589: sensory analysis-general guidance for the design of test rooms. Geneva: International Organisation for Standardization, Geneva, Switzerland.

Jaeger, S. R., Rasmussen, M. A., \& Prescott, J. (2017). Relationships between food neophobia and food intake and preferences: Findings from a sample of New Zealand adults. Appetite, 116, 410-422. doi:10.1016/j.appet.2017.05.030

Johnston, C. A., Palcic, J. L., Tyler, C., Stansberry, S., Reeves, R. S., \& Foreyt, J. (2011). Increasing vegetable intake in Mexican-American youth: A randomized controlled trial. Journal of the American Dietetic Association, 111, 716-720. doi:10.1016/j.jada.2011.02.006

Jordán, J., Tandon, K., Shaw, P., \& Goodner, K. (2001). Aromatic profile of aqueous banana essence and banana fruit by gas chromatography-mass spectrometry (GC-MS) and gas chromatography-olfactometry (GC-O). Journal of Agricultural and Food Chemistry, 49, 4813-4817. doi:10.1021/jf010471k

King, S. C., Meiselman., H. L., \& Henriques, A. (2008). The effect of choice and psychographics on the acceptability of novel flavors. Food Quality and Preference, 19, 692-696. doi:10.1016/j.foodqual.2008.05.003

Knaapila, A., Silventoinen, K., Broms, U., Rose, R. J., Perola, M., Kaprio, J., \& Tuorila, H. M. (2011). Food neophobia in young adults: Genetic architecture and relation to personality, pleasantness and use frequency of foods, and body mass index - A twin study. Behavior Genetics, 41(4), 512-521. doi:10.1007/s10519-010-9403-8

Kort, M., Nijssen, B., van Ingen-visscher, K., \& Donders, J. (2010). Food pairing from the perspective of the 'volatile compounds in food' database. In I. Blank, M. Wüst, \& C. Yeretzian (Eds.), Expression of Multidisciplinary Flavour Science: Proceedings of the 12th Weurman Symposium. Winterthur: Institut of Chemistry and Biological Chemistry.

Krueger, R., \& Casey, M. (2009). Focus groups: A practical guide for applied research. Thousand Oaks, CA: Sage Publications.

Lévy, C. M., MacRae, A., \& Köster, E. P. (2006). Perceived stimulus complexity and food preference development. Acta Psychologica, 123, 394-413. doi:10.1016/j.actpsy.2006.06.006 Mac Con Iomaire, M. (2011). Ireland. In K. Albala (Ed.), Food cultures of the world encyclopaedia. Westport, CT: Greenwood Press.

Mac Con Iomaire, M. (2018). Recognizing food as part of Ireland's intangible cultural heritage. Folk Life, 56(2), 93-115. doi:10.1080/04308778.2018.1502402

Martins, Y., Pelchat, M., \& Pliner, P. (1997). Try it; it's good and it's good for you: Effects of taste and nutrition information on willingness to try novel foods. Appetite, 28(2), 89-102. doi:10.1006/appe.1996.0064 
Martins, Y., \& Pliner, P. (2005). Human food choices: An examination of the factors underlying acceptance/rejection of novel and familiar animal and nonanimal foods. Appetite, 45, 214-224. doi:10.1016/j.appet.2005.08.002

Martins, Y., \& Pliner, P. (2006). “Ugh! That's disgusting!”: Identification of the characteristics of foods underlying rejections based on disgust. Appetite, 46, 75-85. doi:10.1016/j. appet.2005.09.001

Mayr, D., Märk, T., Lindinger, W., Brevard, H., \& Yeretzian, C. (2003). Breath-by-breath analysis of banana aroma by portion transfer reaction mass spectrometry. International Journal of Mass Spectrometry, 223(1-3), 743-756. doi:10.1016/S1387-3806(02)00967-3

Meng, L., David, C. S., \& James, F. T. (1997). Optical chlorophyll sensing system for banana ripening. Postharvest Biology and Technology, 12, 273-283. doi:10.1016/S0925-5214(97) 00059-8

Mustonen, S., Rantanen, R., \& Tuorila, H. (2009). Effect of sensory education on school children's food perception: A 2-year follow-up study. Food Quality and Preference, 20(3), 230-240. doi:10.1016/j.foodqual.2008.10.003

Mustonen, S., \& Tuorila, H. (2010). Sensory education decreases food neophobia score and encourages trying unfamiliar foods in 8-12-year-old children. Food Quality and Preference, 21, 353-360. doi:10.1016/j.foodqual.2009.09.001

Ottenbacher, M. C., \& Harrington, R. J. (2009). The product innovation process of quick-service restaurant chains. International Journal of Contemporary Hospitality Management, 2(5), 522-541.

Park, B.-K., \& Cho, M.-S. (2016). Taste education reduces food neophobia and increases willingness to try novel foods in school children. Nutrition Research and Practice, 10(2), 221-228. doi:10.4162/nrp.2016.10.2.221

Perkel, J. M. (2018). The new molecular gastronomy, or, a gustatory tour of network analysis. Future Science, 53(1), 19-22.

Pliner, P. (1994). Development of measures of food neophobia in children. Appetite, 23(2), 147-163. doi:10.1006/appe.1994.1043

Pliner, P., \& Pelchat, M. L. (1991). Neophobia in humans and the special status of foods of animal origin. Appetite, 16, 205-218. doi:10.1016/0195-6663(91)90059-2

Pliner, P., \& Stallberg-White, C. (2000). "Pass the ketchup, please". Familiar flavors increase children's willingness to taste novel foods. Appetite, 34, 95-103. doi:10.1006/ appe.1999.0290

Raz, C., Piper, D., Haller, R., Nicod, H., Dusart, N., \& Giboreau, A. (2008). From sensory marketing to sensory design: How to drive formulation using consumers' input? Food Quality and Preference, 19(8), 719-726. doi:10.1016/j.foodqual.2008.04.003

Reverdy, C., Chesnel, H., Schlich, P., Köster, E. P., \& Lange, C. (2008). Effect of sensory education on willingness to taste novel food in children. Appetite, 51, 156-165. doi:10.1016/j.appet.2008.01.010

Reverdy, C., Lange, C., \& Schlich, P. (2004, September). Effect of sensory learning on individual sensory performances, perceived complexity of food and consumer preference. In A sense of identity, 1st European conference on sensory science of food and beverages Florence, Italy.

Reverdy, C., Schlich, P., Köster, E., Ginon, E., \& Lange, C. (2010). Effect of sensory education on food preferences in children. Food Quality and Preference, 21(7), 794-804. doi:10.1016/ j.foodqual.2010.03.008

Rozin, E., \& Rozin, P. (1981). Some surprisingly unique characteristics of human food preferences. In A. Fenton \& T. M. Owen (Eds.), Food in Perspective (pp. 243-252). Edinburgh, UK: John Donald Publishers. 
Rozin, P., \& Fallon, A. E. (1986). The acquisition of likes and dislikes for foods, In What is America Eating? Proceedings of a Symposium (pp. 58-71). Washington, D.C.: National Academy Press.

Rozin, P., \& Fallon, A. E. (1987). A perspective on disgust. Psychological Review, 94, 23-41. doi:10.1037/0033-295X.94.1.23

Rozin, P., \& Vollmecke, T. A. (1986). Food likes and dislikes. Annual Review of Nutrition, 6 (1), 433-456. doi:10.1146/annurev.nu.06.070186.002245

Schickenberg, B., van Assema, P., Brug, J., \& De Vries, N. (2008). Are the Dutch acquainted with and willing to try healthful food products? The role of food neophobia. Public Health Nutrition, 11(5), 493-500. doi:10.1017/S1368980007000778

Spence, C., Wang, Q. J., \& Youssef, J. (2017). Pairing flavours and the temporal order of tasting. Flavour, 6(4), 1-15. doi:10.1186/s13411-017-0053-0

Stolzenbach, S., Bredie, W. L. P., \& Byrne, D. V. (2013). Consumer concepts in new product development of local foods: Traditional versus novel honeys. Food Research International, 52(1), 144-152. doi:10.1016/j.foodres.2013.02.030

Stolzenbach, S., Byrne, D. V., \& Bredie, W. L. P. (2011). Sensory local uniqueness of Danish honeys. Food Research International, 44(9), 2766-2774. doi:10.1016/j.foodres.2011.06.006

Tan, H. S. G., Fischer, A. R. H., Tinchan, P., Stieger, M., Steenbekkers, L. P. A., \& van Trijp, H. C. M. (2015). Insects as food: Exploring cultural exposure and individual experience as determinants of acceptance. Food Quality and Preference, 42, 78-89. doi:10.1016/j.foodqual.2015.01.013

Traynor, M. P. (2013). Innovative food product development using molecular gastronomy: A focus on flavour and sensory evaluation (Doctoral dissertation). Retrieved from Arrow@TUDublin. https://arrow.dit.ie/tourdoc/26/

Traynor, M. P., Burke, R., O’Sullivan, M. G., Hannon, J. A., \& Barry-Ryan, C. (2013). Sensory and chemical interactions of food pairings (basmati rice, bacon and extra virgin olive oil) with banana. Food Research International, 54(1), 569-577. doi:10.1016/j. foodres.2013.07.050

Tuorila, H. (1996). Hedonic responses to flavor and their implications for food acceptance. Trends in Food Science \& Technology, 7(12), 453-456. doi:10.1016/S0924-2244(96)10048-0

Tuorila, H. (2007). Sensory perception as a basis of food acceptance and consumption. In H. MacFie (Ed.), Consumer-led food product development (pp. 34-65). Boca Raton, US: CRC Woodhead Publishing.

Tuorila, H., Lähteenmäki, L., Pohjalainen, L., \& Lotti, L. (2001). Food neophobia among the Finns and related responses to familiar and unfamiliar foods. Food Quality and Preference, 12(1), 29-37. doi:10.1016/S0950-3293(00)00025-2

Varshney, K. R., Varshney, L. R., Wang, J., \& Myers, D. (2013). A study in cooking with dirty data. Proceedings of the International Joint Conference on Artificial Intelligence Workshop (pp. 3-12), Beijing, China.

Yeomans, M. R., Chambers, L., Blumenthal, H., \& Blake, A. (2008). The role of expectancy in sensory and hedonic evaluation: The case of smoked salmon ice-cream. Food Quality and Preference, 19(6), 565-573. doi:10.1016/j.foodqual.2008.02.009 